\title{
Luminosity Calibrations with Hipparcos: Theoretical Point of View
}

\author{
Yveline Lebreton \\ Observatoire de Paris, DASGAL, UMR 8633, 92195 Meudon, France
}

\begin{abstract}
Hipparcos provided us with valuable observational material that can be used to anchor more firmly the Galactic and extragalactic distance scales. A calibration which is applicable to all stars must allow for differences in chemical composition and other properties, which may require the use of theoretical models. I discuss some difficulties encountered in the calibration procedure that are related (1) to the determinations of stellar temperatures, luminosities and chemical compositions, (2) to remaining weaknesses of theoretical stellar models, and (3) to the transformations of the model outputs from the $\left(M_{b o l}, T_{\text {eff }}\right)$ plane into any colour-magnitude or colour-colour diagram.
\end{abstract}

\section{Introduction}

Hipparcos provided very accurate distances to many stars located in the solar neighbourhood, including field stars in the disk and in the halo, and members of open clusters. These objects are primary templates towards the Galactic distance scale. They serve to calibrate the distances of more distant or fainter stars and clusters. In these clusters (galactic or globular), bright pulsators (Cepheids or RR Lyrae) are found, the knowledge of their distances being a further fundamental requirement for the assessment of the distance of the Magellanic Clouds or for the building of the extragalactic distance scale.

In any calibration the best-known stars (calibrators) serve to infer the characteristics of their more distant and less well known counterparts, assumed to be governed by the same physical processes. If differences exist (in chemical composition, mass, evolutionary state, rotation, etc.), the calibration process incorporates corrections based on theoretical stellar models or on empirical rules. The accuracy of distance calibration therefore relies on (1) the choice of the best calibrators, (2) the use of secure theoretical stellar models, and (3) the reliability of the conversion of observational data to the input or output parameters of stellar models.

I focus on distance calibrations based on the best-known stars (A-K field dwarfs and subdwarfs and members of the Hyades, Pleiades and Praesepe clusters). The calibration of the distance scale in the Universe has been broadly discussed by Reid (1999), while the impact of Hipparcos results on the stellar structure theory has been reviewed by Lebreton (2000). 


\section{Calibration Tools and Data}

The Hertzsprung-Russell (H-R) diagram analysis requires distances, bolometric fluxes $F_{b o l}$ or magnitudes and bolometric corrections, effective temperatures $T_{\text {eff }}$ or radii, gravities $\log g$ or masses, and abundances of $\mathrm{Fe}, \mathrm{Li}, \alpha$-elements, CNO, etc. Table 1 shows the best present accuracies (see also JD5, these Proceedings) but some data are still uncertain, incomplete or inaccessible: the helium abundance cannot be derived from the spectra of cool stars, abundances are lacking for some elements and some stars (especially subgiants), and very few masses and radii have the desired accuracy of $1-2 \%$.

Stellar models have to be validated with the strong constraints (global parameters, seismology) available for the Sun, nearby single or binary stars, clusters and pulsators. They may then serve to represent (quasi) similar objects.

Observations and stellar models must be properly related. This rests on model atmospheres which fix the external boundary conditions for the interior, and are needed to determine $T_{\text {eff }}, \log g$ and abundances from the spectra and to provide theoretical conversions of the $\left(M_{b o l}, T_{\text {eff }}\right)$ plane to color-magnitude diagrams. Problems with opacities, convection, sphericity or NLTE effects still make the model atmospheres uncertain. As a result $T_{\text {eff-scales may differ by }}$ $200-400 \mathrm{~K}$ and systematic errors in $[\mathrm{Fe} / \mathrm{H}]$ can reach 0.4 dex in metal-poor stars (Gustafsson 1998). Theoretical conversions are therefore still insecure, while empirical calibrations only cover part of the H-R diagram.

Table 1. Best present accuracies for A-K dwarfs and giants calibrators (IRFM: infrared flux method. SBM: surface brightness method)

\begin{tabular}{lllll}
\hline$\sigma_{\pi} / \pi$ & $\sigma_{F_{\text {bol }}} / F_{\text {bol }}$ & $\sigma_{T_{\text {eff }}}(\mathrm{K})$ & $\sigma_{\log g}(\mathrm{dex})$ & $\sigma_{[\mathrm{Fe} / \mathrm{H}]}(\mathrm{dex})$ \\
\hline$<0.10$ & $\sim 0.02$ & $\sim 50-100$ & $\sim 0.1-0.2$ & $\sim 0.05-0.10$ \\
$210^{4}$ stars & $510^{2}$ stars & $10^{3}$ stars & $10^{3}$ stars & $10^{4}$ stars \\
Hipparcos & multi-color & IRFM, SBM & spectroscopy & spectroscopy \\
& photometry & spectroscopy & Hipparcos & photometry \\
\hline \hline
\end{tabular}

\section{Nearby Field Dwarfs: Constraints for Interiors and Atmospheres}

Precise H-R diagrams of the best-known Hipparcos disk and halo stars cannot be fitted with standard stellar models based on the observed metallicities $[\mathrm{Fe} / \mathrm{H}]_{\mathrm{LTE}}$ derived from model atmospheres in LTE: at subsolar metallicities, stars systematically lie on model isochrones computed for $[\mathrm{Fe} / \mathrm{H}]$ higher than $[\mathrm{Fe} / \mathrm{H}]_{\mathrm{LTE}}$ (see Figures 2 and 5 and references in Lebreton 2000).

At low metallicity, UV radiation induces a sizeable overionization of $\mathrm{Fe}$ I (see JD5 and 8). According to Thévenin \& Idiart (1999), as metallicity decreases, this NLTE effect increases the metallicity by increasing amounts : the correction $[\mathrm{Fe} / \mathrm{H}]_{\text {NLTE }}-[\mathrm{Fe} / \mathrm{H}]_{\text {LTE }}$ is $0.0,+0.15$ and $+0.20 \mathrm{dex}$ for $[\mathrm{Fe} / \mathrm{H}]_{\text {LTE }}$-values of $0.0,-1.0$ and -2.0 dex, respectively. Standard stellar models usually neglect microscopic diffusion that transports $\mathrm{He}$ and heavy elements inward resulting in a right shift of the isochrones (Morel \& Baglin 1999; Salaris, Groenewegen, \& Weiss 2000). Models and observations are in good agreement if both the NLTE 

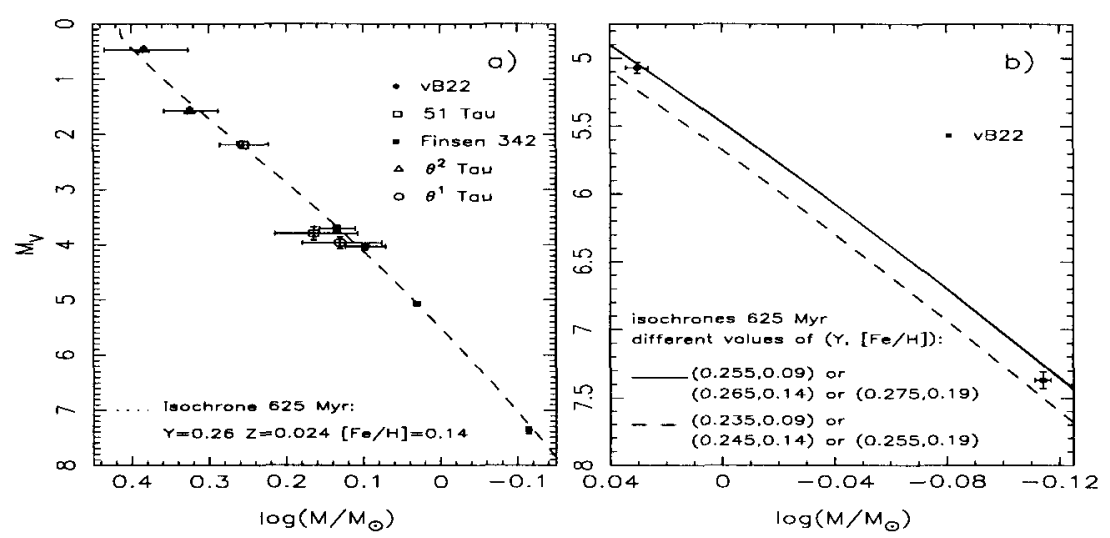

Figure 1. Hyades: mass-luminosity relation

$[\mathrm{Fe} / \mathrm{H}]$-corrections and element diffusion are taken into account when building the isochrones (Lebreton et al. 1999). Remaining uncertainties are related to the dispersion of the $[\alpha / \mathrm{Fe}]$-abundances in halo stars, to the $\sim 0.2-0.3 \mathrm{dex}$ $[\mathrm{Fe} / \mathrm{H}]_{\text {LTE }}$-differences between authors (Sect. 2), and to the unknown efficiency of diffusion. Diffusion is required to explain the He content in the solar convection zone derived from seismology, but hydrodynamical instabilities easily inhibit diffusion and models with diffusion cannot explain some of the Li observations in halo stars.

\section{Open Clusters as First Steps to the Distance Scale in the Universe}

\subsection{The Hyades}

The individual parallaxes based on radial velocities and Hipparcos proper motions are a factor of $\sim 3$ more precise than the trigonometric values (Dravins et al. 1997; de Bruijne, Hoogerwerf, \& de Zeeuw 2000) and yield a mean accuracy $\sigma_{M_{V}} \simeq 0.05 \mathrm{mag}$. The $[\mathrm{Fe} / \mathrm{H}]$-value $(0.14 \pm 0.05 \mathrm{dex})$ is well established (Grenon, these proc.). Isochrones of He-content $Y \simeq 0.26 \pm 0.02$ and age $625 \pm 50$ Myr well fit the main sequence (Lebreton 2000) but the errors in $[\mathrm{Fe} / \mathrm{H}]$ and $T_{\text {eff }}$ limit the precision in Y. The fit is poor at low $T_{\text {eff }}$ because of uncertain colors and model atmospheres. In the turn-off region rotation and overshooting may modify the isochrones while rotation also changes the positions of stars in the $\mathrm{H}-\mathrm{R}$ diagram. Both processes have similar signatures and seismic observations would be required to characterize them. The empirical mass-luminosity relation (Fig. 1) defined by the double-line eclipsing binary vB22 $\left(\sigma_{M} / \mathrm{M}\right.$ and $\sigma_{M_{V}} / M_{V} \lesssim$ $1 \%$ ) allows us to discriminate between the isochrones and to get stronger constraints on $\mathrm{Y}(\Delta \mathrm{Y} \simeq 0.01)$. Other binaries have larger mass error bars $(5-25 \%)$ and provide weaker constraints (Lebreton 2000).

\subsection{Pleiades and Praesepe Clusters}

Mean distances to these clusters were derived from Hipparcos data; there is no evidence of systematic errors in the parallaxes (van Leeuwen, these proc.). Their metallicities are not well known. Spectroscopy gives $[\mathrm{Fe} / \mathrm{H}]=0.10 \pm 0.06 \mathrm{dex}$ 

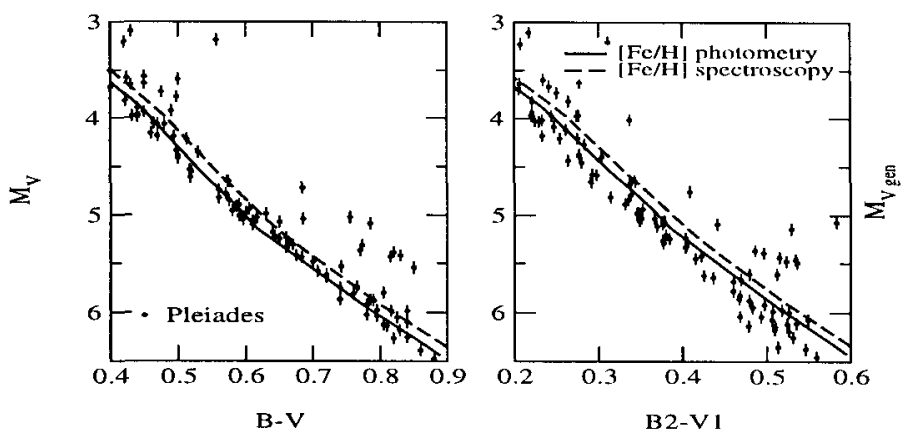

Figure 2. Comparison of model isochrones with the Pleiades data

(Praesepe) and $0.00 \pm 0.05 \mathrm{dex}$ (Pleiades, but values in the range $[-0.03,+0.13]$ can be found in the literature), while Grenon (these proc.) derives respectively $[\mathrm{Fe} / \mathrm{H}]=0.17 \pm 0.01$ and $-0.10 \pm 0.02$ from Geneva photometry. Isochrones calculated with the "photometric" metallicity agree better with observations, in particular in the $\left(M_{V}, B-V\right)$ plane (Fig. 2), but differences in metallicity should be understood. Small adjustments of the helium abundance can improve the fit $\left(\Delta Y=0.02\right.$ corresponds to $\Delta M_{V} \sim 0.06-0.10 \mathrm{mag}$ ) but $\mathrm{Y}$ is constrained by the primordial value and by the value of $\left(Y_{p}<Y \lesssim 0.30\right)$ which has been found for hot stars. Main sequence fitting of isochrones is very sensitive to the models, to their conversion into the observational plane, and to the metallicity. Theoretical isochrones from different authors agree within $\sim 0.05$ mag but the use of different conversions often leads to $M_{V}$ differences as high as $\sim 0.1 \mathrm{mag}$ or more $(0.30 \mathrm{mag})$ ! An error of $0.10 \mathrm{dex}$ on $[\mathrm{Fe} / \mathrm{H}]$ translates into $\sigma_{M_{V}} \sim 0.12-0.15$ mag. In addition colour data are often not homogeneous ( $V-I$ for instance). Any adjustment should ideally provide agreement in all available color indices!

Acknowledgments. I warmly thank Drs. J. de Bruijne, D. Dravins, T. Lejeune, L. Lindegren and N. Robichon who provided observational data.

\section{References}

de Bruijne, J.H.J, Hoogerwerf, R., \& de Zeeuw, P.T. 2000, A\&A, in press Dravins, D., Lindegren, L., Madsen, S., \& Holmberg, J. 1997, ESA SP-402, 733 Gustafsson, B. 1998, in IAU Symp. 189, Fundamental Stellar Properties, ed. T.R. Bedding, A.J. Booth \& J. Davis (Dordrecht: Kluwer), 261

Lebreton, Y. 2000, ARA\&A, 38, 35

Lebreton, Y., Perrin, M.-N., Cayrel, R., Baglin, A., \& Fernandes, J. 1999, A\&A, 350,587

Morel, P. \& Baglin, A. 1999, A\&A, 345, 156

Reid, I.N. 1999, ARA\&A, 37, 191

Salaris, M., Groenewegen, M.A.T. \& Weiss, A. 2000, A\&A, 355, 299

Thévenin, F. \& Idiart, T.P. 1999, ApJ, 521, 753 